Original Research Paper

\title{
Design and Synthesis of Diaminotriazines as Anti- Tuberculosis DHFR Inhibitors
}

\author{
${ }^{1}$ Arundhati C Lele, ${ }^{1}$ Archana Raju, ${ }^{2}$ M.K. Ray, ${ }^{2}$ M.G.R. Rajan and ${ }^{1}$ Mariam S Degani \\ ${ }^{I}$ Department of Pharmaceutical Sciences and Technology, \\ Institute of Chemical Technology, Matunga (E), Mumbai 400019, India \\ ${ }^{2}$ Radiation Medicine Center, Bhabha Atomic Research Centre, \\ Tata Memorial Hospital Annex, Parel, Mumbai-400012, India
}

\author{
Article history \\ Received: 06-11-2014 \\ Revised: $15-11-2014$ \\ Accepted: 31-12-2014 \\ Corresponding Author: \\ Mariam S Degani \\ Department of Pharmaceutical \\ Sciences and Technology, \\ Institute of Chemical \\ Technology, Matunga (E), \\ Mumbai 400019, India \\ Email:ms.degani@ictmumbai.edu.in
}

\begin{abstract}
A series of novel 2,4 diamino-s-triazine was designed as potential Mycobacterium tuberculosis (Mtb) Dihydro folate reductase inhibitors. The synthesized compounds were evaluated in whole cells by employing Resazurin Microtitre Plate Assay (REMA) against Mtb $\mathrm{H}_{37} \mathrm{Rv}$, using known DHFR inhibitors Methotrexate and Trimethoprim as standard drugs. The most active compound in the whole cell assay was selected for DHFR enzyme assay against the pathogen and human enzymes. The enzyme assay results revealed that this compound is a selective pathogenic DHFR inhibitor, with 13 fold more selectivity than Methotrexate. Thus, these derivatives have provided promising selective Hits/Leads as MTb DHFR inhibitors and can provide valuable information for further design of potent anti-TB drugs.
\end{abstract}

Keywords: Mycobacterium Tuberculosis, Dihydrofolate Reductase, Diamino Triazine, Enzyme Inhibition Assay

\section{Introduction}

Tuberculosis (TB) is an airborne, contagious disease caused by Mycobacterium tuberculosis (Mtb) in humans. WHO reported nearly 8.6 million new cases of TB in 2012 including 1.3 million deaths which represent the largest number of incidence of human deaths attributable to a single etiological agent (WHO, 2013). In particular, the current concern is the immense problem resulting from extensively drug-resistant tuberculosis, XDR-TB (Zignol et al., 2006). Dihydrofolate reductase is one of the well validated targets, where more than $1003 \mathrm{D}$-structures are available in Protein Data bank. DHFR is the sole source of tetrahydrofolate and therefore is an Achilles' heel of rapidly proliferating cells (Berman et al., 1991; Schnell et al., 2004). DHFR has been successfully explored as a target for bacterial, protozoal infections (Gangjee et al., 2007) and anti-cancer activities. Only recently, some of the DHFR structures of organisms like Mycobacterium tuberculosis have been explored. Hence there still exists scope for tailoring new molecules as antifolate specifically against $M t b$.

Gerum et al. (2002) have developed a simple and efficient strategy for $M t b$ DHFR inhibitor screening using an engineered strain of Saccharomyces cerevisiae which is dependent on Mtb DHFR for its growth. Using this system, they found that analogs of WR99210 are also potent inhibitors of the Mtb DHFR. Gerum et al. (2002; Li et al., 2000) have reported three-dimensional structure of $M t b$ DHFR. A series of 2,4diaminopyrimidines have been designed and synthesized with a two-carbon tether between a glycerol-mimicking triol and the 6-position of the heterocycle and aryl substituents at the 5-position (El-Hamamsy et al., 2007). Novel boron containing, ortho-icosahedral carborane lipophilic antifolates have also been reported in an attempt to explore carborane-substituted analogs. Kumar et al. (2008) have suggested a structure-based virtual screening workflow for the identification of compounds against $M t b$ DHFR. Recently, a structure-based design of a novel class of potent and selective small peptide inhibitor of $M t b$ DHFR has been reported (Kumar et al., 2010).

Our research group has been involved in the search for DHFR inhibitors. Towards this goal we have reported molecular modeling studies for DHFR inhibitors and also synthesized several classes of novel, diverse inhibitors of DHFR for opportunistic pathogens, including Mycobacterium avium (Bag et al., 2009; Tawari et al., 2011; Bag et al., 2010a; Degani et al., 2010; Bag et al., 2010b). Recently, we have carried out optimization 
studies for isolation of $M t b$ DHFR enzyme from recombinant Saccharomyces cerevisiae (Raju et al., 2013) strains and its purification using a novel affinity column.

Availability of high resolution crystal structures of Mtb DHFR (Li et al., 2000) provides a good starting point for structure-based drug design. In this light, the current work aims at structure-based drug design of potential Mtb DHFR inhibitors.

\section{Materials and Methods}

\section{In-Silico Studies}

An in-silico ADME prediction to check the druggability of the diamino triazine derivatives was performed using QikProp, a program that predicts several significant physical descriptors and relevant pharmaceutical properties of organic molecules (Qikprop, Version 3.5). It provides ranges for comparing the properties of a particular molecule with those of $95 \%$ of known drugs. The descriptors calculated were partition coefficient, Lipinski's rule of five, human oral absorption, CNS activity and gut-blood barrier permeability.

All the designed triazine derivatives were docked on the crystal structure of Mtb DHFR (1DF7), retrived from Brookhaven Protein Databank (www.rcsb.com), to study the in-silico interaction of the designed compounds with the $M t b$ receptor active site. Docking studies were carried out using standard Grid-based Ligand Docking with Energetics (GLIDE) molecular docking protocol implemented within Maestro molecular modelling suite by Schrödinger, LLC, New York, NY, 2006 installed on AMD Athelon workstation (Glide, version 5.0). Initially protein was prepared using protein preparation wizard and grid was generated following which the designed ligands were drawn and cleaned to optimize their energy and geometry (LigPrep, version 3.0). The processed ligands were then docked on to the active site to obtain docking interaction, position and orientation of the designed ligand (ligand pose) with respect to the receptor active site. Docking protocol was validated by reproducing the observed crystallographic structure of the native ligand with a low RMSD value $(0.3 \AA)$ indicating the robustness of the protocol used.

\section{Synthesis}

The Melting points were recorded on Thermomix Compbell electronics, having oil-heating system and were uncorrected. The microwave reactions were carried out using CEM Focused Microwave System in monomode, Model Discover. Analytical Thin-Layer Chromatography (TLC) was carried out on precoated plates $\mathrm{SiO}_{2}$ (Silica gel 60, F 254, Merck). FTIR spectra were recorded on "Buck scientific infrared spectroscopy M500 spectrophotometer" using KBr pellets. NMR spectra were recorded on JEOL AL $300 \mathrm{MHz}$ spectrometer with DMSO-d6 as solvent. All the reagents and chemicals used were of 'reagent grade'. The synthesis of the designed compounds was carried out according to the reactions depicted in Fig. 1.

\section{General Procedure for Synthesis of 2,4- Diamino-s-Triazine}

\section{Step I}

Vanilin $(0.0163 \mathrm{~mol})$, substituted benzyl chloride $(0.0195 \mathrm{~mol})$ and $\mathrm{K}_{2} \mathrm{CO}_{3}(0.018$ moles$)$ was suspended in acetonitrile $(40 \mathrm{~mL})$. Resulting reaction mixture was refluxed for 4-5 h. Reaction was monitored by TLC. After completion of the reaction, as observed from the consumption of the starting material in TLC analysis, reaction mass was filtered to remove $\mathrm{K}_{2} \mathrm{CO}_{3}$, washed with $5 \mathrm{~mL}$ of fresh acetonitrile and the filtrate evaporated to dryness in vacuum to give the corresponding benzyl oxy benzaldehyde derivative 1 in $80-9 \%$ yield.

\section{Step II}

Substituted benzyloxy benzaldehyde derivative $(0.00943 \mathrm{~mol})$, obtained in Step I above was suspended in $30 \mathrm{~mL}$ of acetonitrile and $\mathrm{KOH}$ pellets $(0.00943 \mathrm{~mol})$ were added. Resulting reaction mixture was refluxed for 15-40 min. After completion of reaction, as observed from complete consumption of starting material, reaction mass was concentrated in vacuum and obtained residue was partitioned between water $(25 \mathrm{~mL})$ and ethyl acetate $(25 \mathrm{~mL})$. Organic layer was separated, treated with brine, dried over anhydrous sodium sulphate and adsorbed over silica gel. Desired compound was isolated by column chromatography using hexane:ethyl acetate as mobile phase. Corresponding benzyloxy phenyl acrylonitriles 2 were obtained as crystalline solid in 30-60\% yield.

\section{Step III}

Substituted benzyloxy phenyl acrylonitrile $(0.00211$ mol), obtained in Step II, was suspended in $20 \mathrm{~mL}$ absolute ethanol. Dicyandiamide $(0.00254 \mathrm{~mol})$ was added followed by $\mathrm{KOH}$ pellets $(0.15 \mathrm{~g})$. Resulting reaction mixture was refluxed for 24-40 h. After completion of reaction, as observed from complete consumption of starting material, solid that separated out was filtered at pump. It was further recrystallised using methanol and ethyl acetate mixture. Corresponding 2,4diamino-1,3,5-triazine 3 were obtained as crystalline solid in $40-50 \%$ yield.

\section{Biological Evaluation}

All the synthesized compounds were screened against $M t b \mathrm{H}_{37} \mathrm{Rv}$ using two fold dilution technique, in order to determine the actual Minimum Inhibitory Concentration (MIC) using Resazurin Microtiter Assay (REMA) (Palomino et al., 2002). 


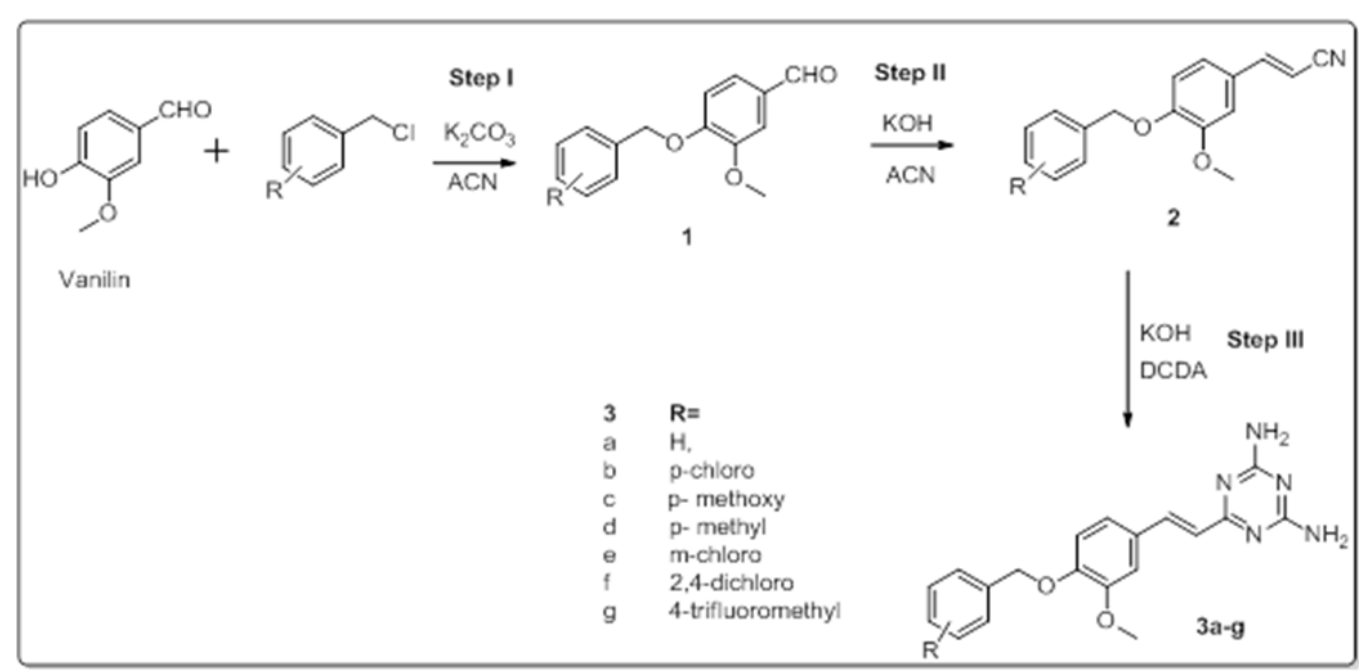

Fig. 1. Synthesis of diamino triazine derivatives

Table 1. Qikprop analysis results of designed diamino triazine derivatives along with that of Methotrexate and Trimethoprim

\begin{tabular}{|c|c|c|c|c|c|c|c|}
\hline Comp. & $\mathrm{MW}^{\mathrm{a}}$ & \#Stars ${ }^{\mathrm{b}}$ & $\mathrm{CNS}^{\mathrm{c}}$ & $\mathrm{QP} \log \mathrm{Po} / \mathrm{w}^{\mathrm{d}}$ & QPP Caco ${ }^{\mathrm{e}}$ & $\% \mathrm{HOA}^{\mathrm{f}}$ & Rule of Five ${ }^{\mathrm{g}}$ \\
\hline$\overline{3 a}$ & 349.39 & 0 & -2 & 2.80 & 199.82 & 84.56 & 0 \\
\hline $3 b$ & 383.83 & 0 & -2 & 3.27 & 199.82 & 87.28 & 0 \\
\hline $3 \mathrm{c}$ & 379.41 & 0 & -2 & 2.91 & 199.82 & 85.19 & 0 \\
\hline $3 d$ & 363.41 & 0 & -2 & 3.09 & 199.82 & 86.23 & 0 \\
\hline $3 \mathrm{e}$ & 383.83 & 0 & -2 & 3.27 & 199.82 & 87.28 & 0 \\
\hline $3 f$ & 418.28 & 0 & -2 & 3.69 & 199.88 & 89.73 & 0 \\
\hline $3 g$ & 417.39 & 0 & -2 & 3.79 & 199.88 & 90.34 & 0 \\
\hline MTX & 454.44 & 4 & -2 & -1.80 & 0.05 & 0.00 & 2 \\
\hline TMP & 290.32 & 0 & -2 & 0.91 & 391.62 & 78.73 & 0 \\
\hline
\end{tabular}

${ }^{a}$ Molecular weight-acceptable limit is $<500 \mathrm{Da}$

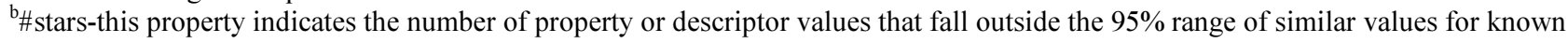
drugs. A large number of stars suggest that a molecule is less drug-like than molecules with few stars. The range predicted for this parameter using QikProp is 0-5; where 0 indicates no violation or best candidate

${ }^{\mathrm{C}} \mathrm{CNS}$-this exhibits the predicted central nervous system activity on a -2 (inactive) to +2 (active) scale

${ }^{\mathrm{d}} \mathrm{QP} \log \mathrm{Po} / \mathrm{w}$-this gives the predicted octanol/water partition coefficient. The acceptable range predicted for this parameter using QikProp is- 2.0 to 6.5

${ }^{\mathrm{e}}$ QPPcaco-this gives the predicted apparent Caco-2 cell permeability in $\mathrm{nm} / \mathrm{s}$. Caco-2 cells are a model for the gut-blood barrier. QikProp predictions are for non-active transport, where $<25$ is considered poor and $>500$ is considered excellent

${ }^{f}$ Percentage human oral absorption-this gives the predicted human oral absorption on $0-100 \%$ scale. The prediction is based on a quantitative multiple linear regression model. Value of $>80 \%$ is considered good and $<25 \%$ is considered poor

${ }^{\mathrm{g}}$ Rule of five-this property denotes the number of violations of Lipinski's rule of five. The rules includes: $\mathrm{MW}<500, \mathrm{QP} \operatorname{logPo} / \mathrm{w}<5$, donor $\mathrm{HB} \leq 5$, accpt $\mathrm{HB} \leq 10$. Compounds that satisfy these rules are considered drug like

MIC is the lowest concentration at which complete inhibition was observed and was determined by visual inspection (color change from blue to pink in case of growth) (Table 2). Methotrexate (MTX) and Trimethoprim (TMP) were used as reference drugs

\section{Enzyme Assay}

The most active derivative, in the whole cell assay, was subjected to enzyme assay against the $M t b$ and human DHFR enzymes. The activity was determined spectrophotometrically (White et al., 2004) by monitoring the decreasing absorbance for two mins at $340 \mathrm{~nm}$ and $37^{\circ} \mathrm{C}$. Standard assay mixture contained 50 $\mathrm{mM}$ potassium phosphate ( $\mathrm{pH} 7.4), 5 \mathrm{mM}$ DTT and 60 $\mu \mathrm{M}$ NADPH and enzyme. The reaction was started after 3 min of incubation by the addition of $45 \mu \mathrm{M}$ DHF. A unit of the enzyme activity is defined as the amount of the enzyme converting $1.0 \mu \mathrm{mol}$ of DHF and NADPH to THF and NADP ${ }^{+}$per min. This is calculated from the change in absorbance at $340 \mathrm{~nm}$ under specified assay condition (Hillcoat et al., 1967).

\section{Results}

\section{In-Silico Screening}

QikProp analysis of diamino triazine analogs (Table 1) indicated that all the designed molecules have drug-like properties. 


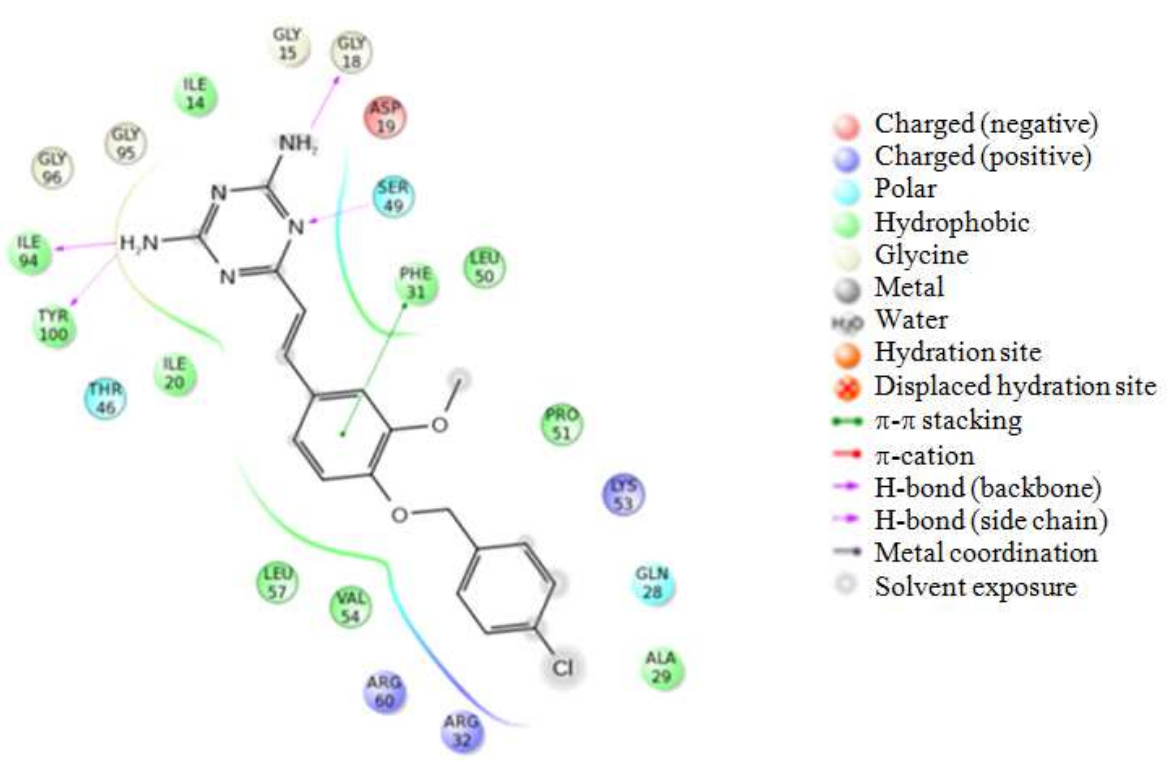

Fig. 2. Docking interaction of $3 \mathrm{~b}$ with $M t b$ DHFR active site

Table 2. Whole cell assay of the synthesize derivatives against $M t b \mathrm{H}_{37} \mathrm{Rv}$

\begin{tabular}{llr}
\hline Comp. & $\mathrm{R}=$ & $\mathrm{MIC} \mu \mathrm{g} / \mathrm{mL}$ \\
\hline $3 \mathrm{a}$ & $\mathrm{H}$ & 70.00 \\
$3 \mathrm{~b}$ & $4-\mathrm{Cl}$ & 126.00 \\
$3 \mathrm{c}$ & $4-\mathrm{OCH}_{3}$ & 125.00 \\
$3 \mathrm{~d}$ & $4-\mathrm{CH}_{3}$ & 62.50 \\
$3 \mathrm{e}$ & $3-\mathrm{Cl}$ & 70.00 \\
$3 \mathrm{f}$ & $2,4 \mathrm{diCl}$ & 8.90 \\
$3 \mathrm{~g}$ & $4-\mathrm{CF}_{3}$ & 15.62 \\
MTX & - & 0.44 \\
TMP & - & 8.40 \\
\hline
\end{tabular}

Table 3. Enzyme assay of $3 \mathrm{f}$ against $M t b$ and human DHFR

\begin{tabular}{llll}
\hline Comp. & $\mathrm{IC}_{50}$ (Mtb DHFR) $\mu \mathrm{M}$ & $\mathrm{IC}_{50}$ (Human DHFR) $\mu \mathrm{M}$ & Selectivity \\
\hline 3f & $625 \pm 0.043$ & $1575 \pm 0.0062$ & 2.520 \\
MTX & $0.00825 \pm 0.00025$ & $0.0016 \pm 0.0003$ & 0.194 \\
TMP & $16.09 \pm 0.32$ & $83.46 \pm 3.46$ & 5.187 \\
\hline
\end{tabular}

All the designed diamino triazines were successfully docked in the Mtb DHFR active site. Necessary docking interactions were observed with important residues of the enzyme active site.

Docking interactions of $3 \mathrm{~b}$ have been depicted in Fig. 2.

\section{Biological Screening}

The synthesized derivatives were tested against Mycobacterium tuberculosis $\mathrm{H}_{37} \mathrm{Rv}$ strain using Resazurin microtitre assay. REMA is a type of broth dilution method employing Resazurin dye as a growth indicator wherein the dye gets reduced to Resazurin in presence of viable organisms, giving a colour change from blue to pink. Reported DHFR inhibitors Methotrexate and Trimethoprim have been used as reference standards. The details of the activity obtained have been given in Table 2.

\section{Enzyme Assay}

The most potent derivative was tested for its ability to inhibit Mtb DHFR enzyme. Further, to evaluate the selectivity of the compound towards Mtb DHFR over human DHFR, enzyme assay using human DHFR was also carried out. MTX and TMP were used as antifolate standards. The enzyme assay results have been shown in Table 3.

\section{Discussion}

\section{In-Silico Screening}

From the in-silico ADME analysis of the designed diamino triazine analogs, it was observed that the designed inhibitors exhibited excellent drug likeliness. Most of the molecules exhibited physicochemical properties which fall in the range of known drugs. 
All the designed diamino triazines showed favourable binding interactions with crucial active site residues of $M t b$ DHFR. The amino group of the triazine ring is involved in H-bond interaction with the hydrophilic residues at the bottom of the active site cavity while the distal aromatic ring is involved in hydrophobic interactions with residues at the mouth of the active site tunnel. Additional $\pi-\pi$ stacking interactions were observed in many derivatives, with residue Phe 31.

\section{Synthesis}

Retrosynthetic analysis was carried out and suitable synthetic scheme was planned depending on the basis of synthetic feasibility and availability of starting materials. Substituted benzyl chlorides were reacted with vanillin, in presence of $\mathrm{K}_{2} \mathrm{CO}_{3}$, to give the corresponding 1a-g intermediates. Purification was carried out by filtration and solvent treatment. Further these intermediates were condensed with acetonitrile to give $\alpha-\beta$ unsaturated nitrile intermediates $2 \mathrm{a}-\mathrm{g}$. These nitrile intermediates were cyclized were converted to target compounds, 3a-g, using dicyandiamide, $\mathrm{KOH}$ and ethanol as solvent. The synthesized compounds were suitably characterized.

\section{Biological Evaluation}

The in-vitro whole cell activity evaluation against Mtb $\mathrm{H}_{37} \mathrm{Rv}$ indicated that the unsubstituted parent compound, 3a, has activity of $70 \mu \mathrm{g} \mathrm{mL}$, giving a good start point for further modifications. Substitution at the meta position does not affect the activity. Substitution at the para position leads to decrease in the activity, with the exception of the trifluoro methyl group. The better activity of the trifluoro methyl group could be because of some additional interactions of the fluorine atoms with the receptor active site. The most potent derivative was found to be the $2,4 \mathrm{di} \mathrm{Cl}$ substituted analog. Substitution at the ortho position might play a key role in activity enhancement.

\section{Enzyme Asssay}

Compound 3f has exhibited promising antituberculosis activity in whole cell assay was tested for its ability to inhibit $M t b$ and human DHFR enzyme. The enzyme assay indicates that the compound is moderately active, yet selective towards the pathogenic enzyme. It is 13 fold more selective than Methotrexate, which is a very potent but highly toxic known DHFR inhibitor.

\section{Conclusion}

The results indicate that these derivatives show promising anti-tuberculosis activity. Of particular note is compound $3 \mathrm{f}$ which has shown activity comparable to Trimethorpim, which is 5 times more selective towards Mtb DHFR while Methotrexate is very active yet highly toxic and has been widely used as anti- cancer agent. The enzyme assay results reveal that the most active compound $3 \mathrm{f}$ is moderately active yet 13 fold more selective than Methotrexate. Taking into account our preliminary results, our efforts are now focused on further development of diamino triazines as promising $M t b$ DHFR inhibitors.

\section{Acknowledgement}

Author Arundhati Lele is thankful to Council of Scientific and Industrial Research, New Delhi and Archana Raju is thankful to Indian Council of Medicinal Research, New Delhi for financial assistance.

\section{Author's Contributions}

All authors equally contributed in this work.

\section{Ethics}

This article is original and contains unpublished material. The corresponding author confirms that all of the other authors have read and approved the manuscript and no ethical issues involved.

\section{References}

Bag, S., N.R. Tawari and M.S. Degani, 2009. Insight into inhibitory activity of mycobacterial dihydrofolate reductase inhibitors by in-silico molecular modeling approaches. QSAR Combinatorial Sci., 28: 296-311.

DOI: 10.1002/qsar.200860067

Bag, S., N.R. Tawari, M.S. Degani and S.F. Queener, 2010a. Design, synthesis, biological evaluation and computational investigation of novel inhibitors of dihydrofolate reductase of opportunistic pathogens. Bioorganic Med. Chemistry, 18: 3187-3197. DOI: $10.1016 /$ j.bmc.2010.03.031

Bag, S., N.R. Tawari, S.F. Queener and M.S. Degani, 2010b. Synthesis and biological evaluation of biguanide and dihydrotriazine derivatives as potential inhibitors of dihydrofolate reductase of opportunistic microorganisms. J. Enzyme Inhibition Med. Chem., 25: 331-339. DOI: $10.3109 / 14756360903179443$

Berman, E.M. and L.M. Werbel, 1991. The renewed potential for folate antagonists in contemporary cancer chemotherapy. J. Med. Chem., 34: 479-485. DOI: $10.1021 / \mathrm{jm} 00106 \mathrm{a} 001$

Degani, M.S., S. Bag, R. Bairwa, N.R. Tawari and S.F. Queener, 2010. Novel 2-hydrazino-pyrimidin-4(3H)one derivatives as potential dihydrofolate reductase inhibitors. J. Heterocyclic Chem., 47: 558-563. 
El-Hamamsy, M.H., A.W. Smith, A.S. Thompson and M.D. Threadgill, 2007. Structure-based design, synthesis and preliminary evaluation of selective inhibitors of dihydrofolate reductase from Mycobacterium tuberculosis. Bioorganic Med. Chem., 15: 4552-4576.

DOI: $10.1016 /$ j.bmc.2007.04.011

Gangjee, A., S. Kurup and O. Namjoshi, 2007. Dihydrofolate Reductase as a target for chemotherapy in parasites. Current Pharmaceutical Design, 13: 609-639.

DOI: $10.2174 / 138161207780162827$

Gerum, A.B., J.E. Ulmer, D.P. Jacobus, N.P. Jensen and D.R. Sherman et al., 2002. Novel Saccharomyces cerevisiae screen identifies WR99210 analogues that inhibit Mycobacterium tuberculosis dihydrofolate reductase. Antimicrobial Agents Chemotherapy, 46: 3362-3369. DOI: 10.1128/AAC.46.11.3362-3369

Hillcoat, B.L., P.F. Nixon and R.L. Blakley, 1967. Effect of substrate decomposition on the spectrophotometric assay of dihydrofolate reductase. Analytical Biochem., 21: 178-189. DOI: 10.1016/0003-2697(67)90179-0

Kumar, A. and M.I. Siddiqi, 2008. Virtual screening against Mycobacterium tuberculosis dihydrofolate reductase: Suggested workflow for compound prioritization using structure interaction fingerprints. J. Molecular Graphics Modell., 27: 476-488. DOI: 10.1016/j.jmgm.2008.08.005

Kumar, M., R. Vijayakrishnan and G.S. Rao, 2010. In silico structure-based design of a novel class of potent and selective small peptide inhibitor of Mycobacterium tuberculosis Dihydrofolate reductase, a potential target for anti-TB drug discovery. Molecular Diversity, 14: 595-604.

DOI: $10.1007 /$ s 1 1030-009-9172-6

Li, R., R. Sirawaraporn, P. Chitnumsub, W. Sirawaraporn and J. Wooden et al., 2000. Threedimensional structure of $M$. tuberculosis dihydrofolate reductase reveals opportunities for the design of novel tuberculosis drugs. J. Molecular Biol., 295: 307-323.

DOI: $10.1006 /$ jmbi. 1999.3328
Palomino, J.C., A. Martin, M. Camacho, H. Guerra and J. Swings et al., 2002. Resazurin microtiter assay plate: Simple and inexpensive method for detection of drug resistance in Mycobacterium tuberculosis. Antimicrobial Agents Chemotherapy, 46: 2720-2722. DOI: $10.1128 /$ AAC.46.8.2720-2722.2002

Raju, A., M.S. Degani, M.A. Khedkar and S.A. Niphadkar, 2013. Novel affinity chromatographic technique for purification of dihydrofolate reductase from recombinant yeast. Int. J. Drug Design Discovery, 4: 994-997.

Schnell, J.R., H.J. Dyson and P.E. Wright, 2004. Structure, dynamics and catalytic function of dihydrofolate reductase. Ann. Rev. Biophys. Biomolecular Structure, 33: 119-140.

DOI: 10.1146/annurev.biophys.33.110502.133613

Tawari, N.R., S. Bag and M.S. Degani, 2011. A review of molecular modelling studies of dihydrofolate reductase inhibitors against opportunistic microorganisms and comprehensive evaluation of new models. Curr. Pharmaceutical Design, 17: 712-751. DOI: $10.2174 / 138161211795428966$

White, E.L., L.J. Ross, A. Cunningham and V. Escuyer, 2004. Cloning, expression and characterization of Mycobacterium tuberculosis dihydrofolate reductase. FEMS Microbiol. Lett., 232: 101-105. DOI: $10.1016 / \mathrm{S} 0378-1097(04) 00038-2$

WHO, 2013. Global tuberculosis report 2013. World Health Organization, Glide, Version 5.0, Schrödinger, LLC, New York.

Zignol, M., M.S. Hosseini, A. Wright, C.L. Weezenbeek and P. Nunn et al., 2006. Global incidence of multidrug-resistant tuberculosis. J. Infec. Dis., 194: 479-485. DOI: $10.1086 / 505877$ 